\title{
A practical inspiration on used product recycling in Chinese context: recovery-responsibility trading right strategy
}

\author{
NIU Shuiye ${ }^{a}$, XU Fangchaob \\ Business School, Nankai University, Tianjin 300071, PR China \\ ablueanglelove@126.com, ${ }^{b}$ xufc@mail.nankai.edu.cn
}

\begin{abstract}
Keywords: used product recycling; Chinese context; recovery responsibility; trading right.
Abstract. Faced with the international environmental pressure and transaction obstacles, Chinese government has enacted and implemented laws which contain the requirement of EPR (Extended Producer Responsibility) in manufacturing industry. However, the implementation effect is not ideal. Firstly, this paper explores the current characteristics of used product recycling in Chinese manufacturing industry via the method of case study: a. the recycling condition of OEM-dominated mode is relatively mature for many $O E M \mathrm{~s}$ which have established their own recycling subsidiary or held shares in third party recycling enterprises; b. the existing $O E M$ recycling capacity is uneven and not furthest utilized; c. the $O E M s^{\prime}$ initial EPR efforts are not effectively stimulated and rewarded. Based on the above features, the recovery-responsibility trading right of used product recycling is put forward. Compared to the carbon trading right, the recovery-responsibility trading right emphasizes some special factors. This paper systematically elaborates the idea of recoveryresponsibility trading right strategy and proposes the implementation framework of recoveryresponsibility trading right strategy based on the practical operation.
\end{abstract}

\section{Introduction}

In recent years, with rapid economic growth and technological development, the quantity of used products in China is ever-increasing at a high speed. According to a forecast report of Chinese electrical and electronic industry, in 2020, the number of discarded mobile phones will reach seven times of the amount in the early years of the 21 st century. Sustainable development objectives and legislation regarding the environment stimulate manufacturing enterprises to collect and recycle end-of-life products as well as reducing waste during production, which embodies the main content of EPR (Extended Producer Responsibility) practices ${ }^{[1]}$. Nowadays, producers must take their products' whole life environment responsibility under EPR. Some famous companies, such as $B M W$, Volkswagen, Honda, Xerox, Kodak, have taken on the business operation of end-of-life products recycling. However, in China, the overall situation on used product recycling is not ideal. Our research focuses on the Chinese dilemma and puts forward a new strategy to deal with this problem.

Characteristics of Chinese Used Product Recycling. Recently, some remarkable changes have taken place in Chinese OEMs (Original Equipment Manufacturers). Firstly, OEMs consider not only economic benefit but also environmental and social benefits when making their production decisions. Secondly, many OEMs establish their own recycling subsidiary or hold shares in third party recycling enterprise, such as China FAW Group; Anhui Changjiang Auto Group Co., Ltd., Chery Automobile Co., Shanghai Volkswagen United Development Co., Ltd. , Weichai Power (Weifang) and Manufacturing Co. Ltd., etc. in the automobile remanufacture field. Thirdly, OEMs must govern the whole supply chain based on EPR concept to deal with the competition in closedloop supply chain. At the same time, Chinese government carries out two policies: charging the recovery and recycling fee for OEMs' every unit original product and giving subsidies for $3 P / O E M$ remanufacturers' every recycled product.

Under the joint efforts of the Chinese government and $O E M S$, China's used product recycling market highlights the following key characteristics: a. the existing recycling capacity of $O E M s$ is uneven and not furthest utilized while the operation condition of OEM-dominated mode is relatively mature. b. the $O E M s^{\prime}$ efforts on the use of green materials and design for remanufacturing are not 
effectively stimulated and rewarded, while the reward and punishment measures are used for a relatively long time.

Thus, the current operation mode of used product recycling in China faces a huge challenge. It is urgent for Chinese government to change the strategy-thinking perspective and design a more effective and applicable strategy scheme. There is no doubt that the above practical analysis and judgment of China's actual situation can provide a directional guidance.

Formation of Recovery-responsibility Trading Right Strategy. This section states the origin and the connotation of recovery-responsibility trading right strategy.

Origin of the Strategy Thought. The traditional view of used product recycling is a kind of producer's extended responsibility. This view fundamentally influences producer's enthusiasm on participating in the recycling practice. Thus, converting the cognitive angle of view is worthy of attention. Is it feasible to make the used product recycling to be a kind of right for producers? Can we improve the occasion of Chinese used product recycling? These two essential questions make us come up with the idea of recovery-responsibility trading. This strategy emphasizes the principles of "enterprise autonomy before government governance" and "source control". The motivation or goal of this idea is to stimulate the enthusiasm and initiative of recycling, as well as exploit the handling capacity of $O E M s$ themselves.

The Connotation of Recovery-responsibility Trading Right. Similar to carbon trading right ${ }^{[2]}$, the recovery-responsibility trading right is also a quota assigned by the Chinese government on $O E M$ 's recovery responsibility. One difference is that the government looks forward to increased rather than reduced recovery-responsibility intensity year by year. When an $O E M$ 's recovery quota is less than its usable recycling capacity, it gets the "right" to sale the extra capacity to other $O E M s$ whose recovery quota are beyond their potential recycling capacity. When a company purchases the recovery-responsibility quota, its own used product recycling pressure is reduced accordingly. Another difference between the carbon quota and recovery-responsibility quota fills in the calculation formula (see Eq.1 and Eq.2), emphasizing the role of $I M E$ in the latter's calculation.

$$
\begin{aligned}
& C Q=C I \times I A V . \\
& R Q=R I \times I A V / I M E .
\end{aligned}
$$

$C Q, R Q$ denote the carbon quota, the recovery-responsibility quota; $C I, R I$ denote the carbon intensity, the recovery-responsibility intensity; IAV denotes the industrial added value; IME denotes the degree of $O E M s^{\prime}$ initial manufacturing efforts, including the use of green materials, design for remanufacturing, etc.

Operation Framework of Recovery-responsibility Trading Right Strategy. The main operation framework of the recovery-responsibility trading right strategy includes two main processes: the deployment process, the trading and governance process. This section clarifies the detailed process of implementing this strategy.

The Deployment Process of Recovery-responsibility Quota. On the basis of national industry classification, the clustering analysis is firstly implemented for different $O E M s$ in a certain industry. In a broad category, all the OEMs are divided into the large group and the small and medium-sized group according to different $O E M s^{\prime}$ ' expected $R I$ sizes. The data analysis of last three-year operation is done for all enterprises in a certain group. The special analysis content includes the historical amount of used products, the industrial added value, the historical $R I$, etc. After data processing, the weighted average $R I$ baseline is obtained as the ratio of all enterprises' annual recycling quantity and all enterprises' annual industrial added value. And based on the above data, the $R I$ baselines in recent three years can be determined. According to the data of historical $R I$ baselines and the government's $R I$ increase rate standard, the targeted $R I$ baselines in the next three years can be calculated out. Further, according to Eq.2, the total distributable quota of the certain group in the next three years can be obtained ${ }^{[3]}$. All $O E M s$ in the certain group participate in the $R Q$ game via national declaration system. The calculation of an $O E M$ 's submitted $R Q$ also refers to the $E q .2$.What needs to be emphasized is that it must conform to the government's requirements of the 
biggest growth of used product quantity, the lowest $R I$, and the differential RI increase rate. Ultimately, the government adjusts every $O E M$ 's $R Q$ and determines the targeted $R I$ and $R Q$ in the next three years for every $O E M$. The government adjusts the targeted $R I$ and $R Q$ once again in the progress of practice. A concise display of the whole process is shown in Fig.1.

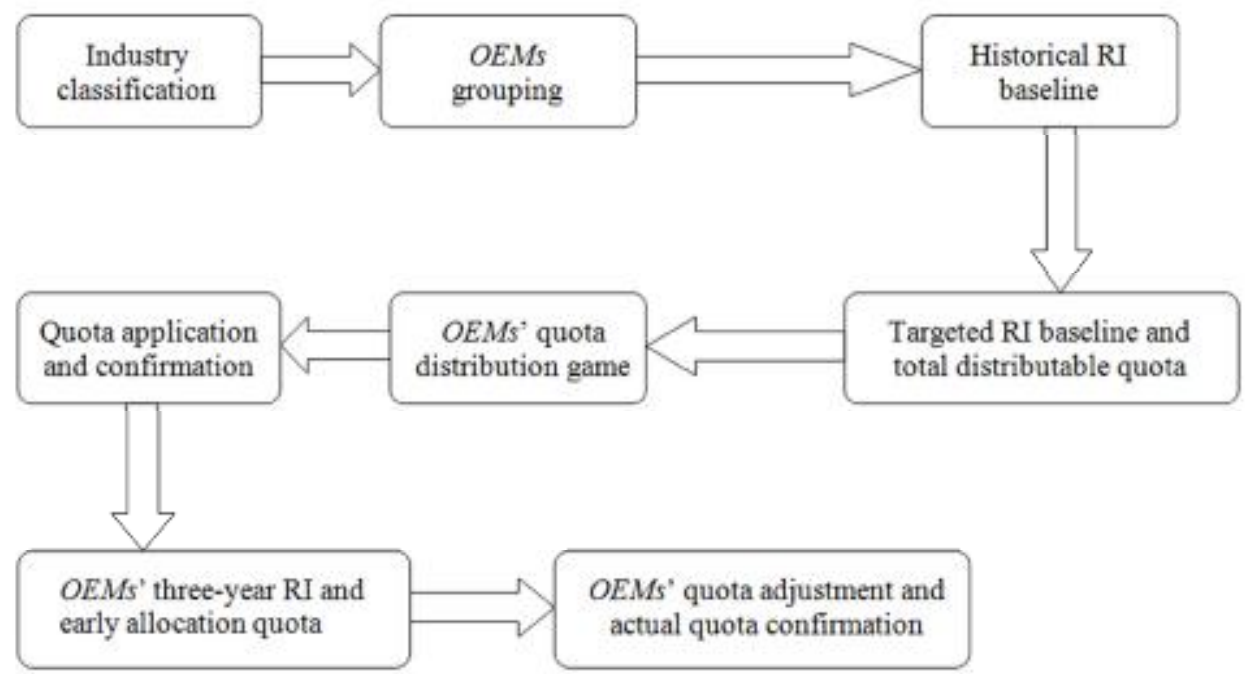

Fig. 1. The deployment process of recovery-responsibility quota.

The Trading and Governance Process of $O E M$ 's Recovery-responsibility. In this process, the government, OEMs and 3P-recycler need to cooperate with each other. Let $\alpha_{i}\left(\alpha_{j}\right), \gamma_{i}\left(\gamma_{j}\right), \Delta \gamma_{i}\left(\Delta \gamma_{j}\right)$, $q_{i}\left(q_{j}\right), \mathrm{Q}_{i}\left(\mathrm{Q}_{j}\right), \mathrm{K}_{i}\left(\mathrm{~K}_{j}\right), P_{i}\left(P_{j}\right), C_{r i}\left(C_{r j}\right), C_{m i}\left(C_{m j}\right), \pi_{i}\left(\pi_{j}\right)$ denote the $O E M_{i}$ 's $\left(O E M_{j}\right.$ 's $) I M E, R I$, expected added $R I, R Q$, total original product production quantity, total recycling capacity of used products, unit original product sale price, unit used product recycling cost, unit original product manufacture cost, expected profit. Let $\beta_{i j}, y_{i j}, p_{i j}$ denote the used product exchange ratio, the trading $R Q$ quantity, the unit trading price when the $O E M_{i}$ makes transaction with the $O E M_{j}$. We can define one or more parameters as variables to construct a game model to maximize every participant's expected profit ${ }^{[4,5]}$.

The buyer $\left(O E M_{i}\right)$ expected profit:

$$
\begin{aligned}
& \pi_{i}=\left(P_{i}-C_{m i}\right) \mathrm{Q}_{i}-C_{r i}\left[\frac{\left(\gamma_{i}+\Delta \gamma_{i}\right)\left(P_{i}-C_{m i}\right) \mathrm{Q}_{i}}{\alpha_{i}}-y_{i j}\right]-p_{i j} y_{i j} . \\
& \text { S.t. } \frac{\left(\gamma_{i}+\Delta \gamma_{i}\right)\left(P_{i}-C_{m i}\right) \mathrm{Q}_{i}}{\alpha_{i}}-y_{i j} \leq \mathrm{K}_{i}
\end{aligned}
$$

The seller $\left(O E M_{j}\right)$ expected profit:

$$
\begin{aligned}
& \pi_{j}=\left(P_{j}-C_{m j}\right) \mathrm{Q}_{j}-C_{r j}\left[\frac{\left(\gamma_{j}+\Delta \gamma_{j}\right)\left(P_{j}-C_{m j}\right) \mathrm{Q}_{j}}{\alpha_{j}}+\beta_{i j} y_{i j}\right]+p_{i j} y_{i j} \cdot \\
& \text { S.t. } \frac{\left(\gamma_{j}+\Delta \gamma_{j}\right)\left(P_{j}-C_{m j}\right) \mathrm{Q}_{j}}{\alpha_{j}}+\beta_{i j} y_{i j} \leq \mathrm{K}_{j}
\end{aligned}
$$

As for the untreated used products, the government adopts the unified management mechanism. The government evaluates and classifies the different recyclers' levels: high, medium, or low, then awards the recycling qualification and assigns the recycling product category and quantity. To stimulate 3P-recyclers, the national government should give them corresponding subsidies according to the product processing difficulty. Assume that there are three enterprises in the system. The $O E M c$ wants to trade with $O E M_{A}, O E M_{B}$, and $\alpha_{A}>\alpha_{B}>\alpha_{C}$. The detailed operation can be seen in Fig. 2. 


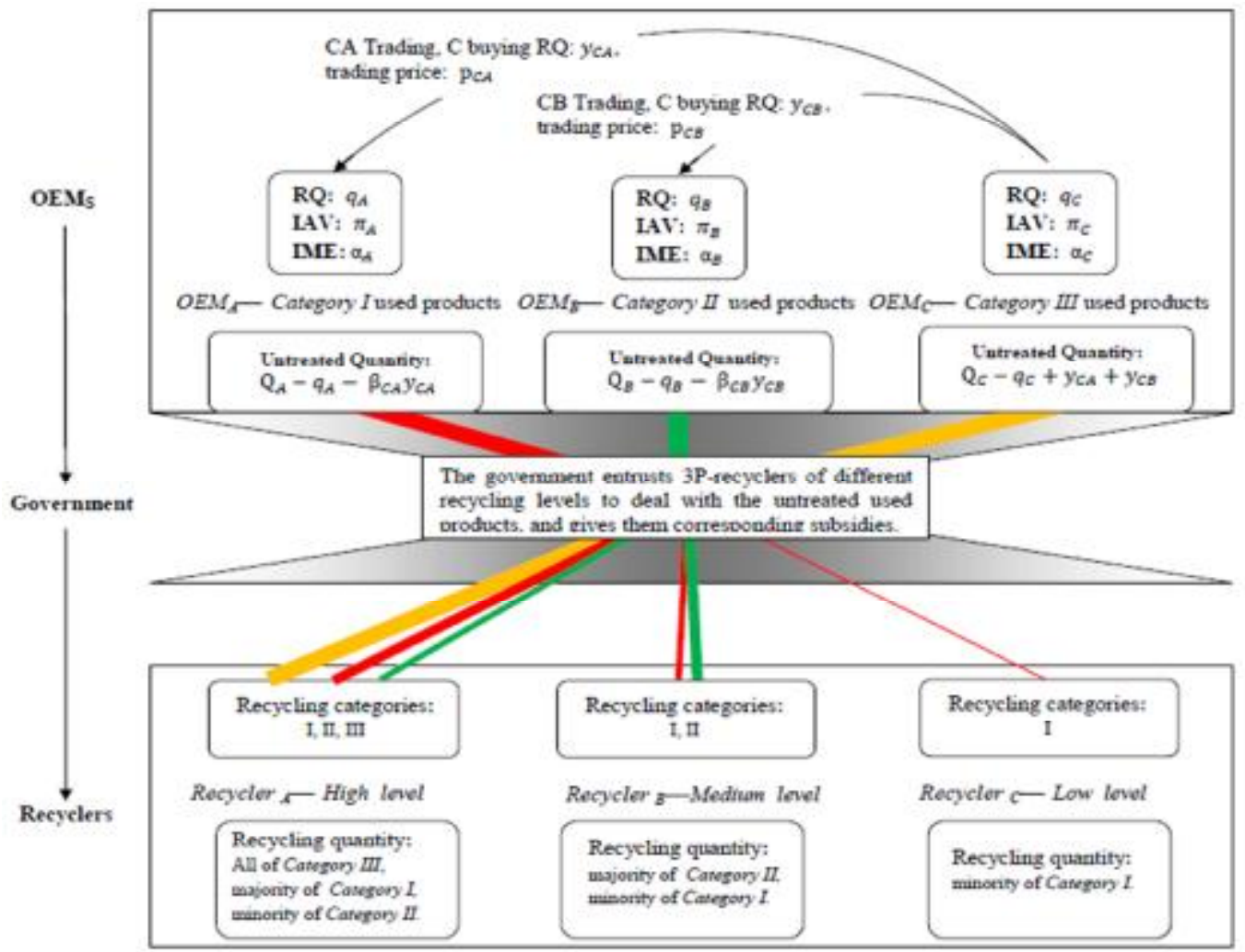

Fig. 2. The trading and governance process of $O E M$ 's recovery-responsibility.

\section{Conclusions}

This paper detailedly analyzes the current characteristics of used product recycling in Chinese manufacture industries. In this Chinese context, this study puts forward a new strategy: carrying out recovery-responsibility trading right. This paper systematically elaborates the idea of this strategy, and constructs the implementation framework of recovery-responsibility trading right strategy based on the practical operation. Besides, some equations are used to describe the key contents. Conducting a game model to explore the optimal trading and production decisions can be a meaningful future research direction.

\section{Acknowledgements}

This work was financially supported by the Major Program of National Social Science Fund of China (Grant No. 13\& ZD147).

\section{References}

[1] Lifset, Reid, Atalay Atasu, and Naoko Tojo. "Extended producer responsibility." Journal of Industrial Ecology 17.2 (2013): 162-166.

[2] Johnson, Eric, and Russell Heinen. "Carbon trading: time for industry involvement." Environment International 30.2 (2004): 279-288.

[3] Raupach, Michael R., et al. "Sharing a quota on cumulative carbon emissions." Nature Climate Change 4.10 (2014): 873-879.

[4] Ling, Li, and Deng Feiqi. "Equilibrium solution of two enterprises cooperative game." Journal of Systems Engineering and Electronics 18.2 (2007): 270-274.

[5] Faccio, Maurizio, et al. "Sustainable SC through the complete reprocessing of end-of-life products by manufacturers: A traditional versus social responsibility company perspective." European Journal of Operational Research 233.2 (2014): 359-373. 